\title{
Intravascular CNS lymphoma: Successful therapy using high-dose methotrexate-based polychemotherapy
}

\author{
Sied Kebir ${ }^{1}$, Klaus Kuchelmeister ${ }^{3}$, Pitt Niehusmann ${ }^{3}$, Michael Nelles², Young Kim², Sharmilan Thanendrarajan ${ }^{4}$, \\ Niklas Schäfer ${ }^{1}$, Moritz Stuplich', Frederic Mack', Björn Scheffler ${ }^{5}$, Horst Urbach², Martin Glas ${ }^{1,5}$ \\ and Ulrich Herrlinger ${ }^{1 *}$
}

\begin{abstract}
Intravascular diffuse large B-cell lymphoma limited to the CNS (CIVL) is a very rare malignant disorder characterized by a selective accumulation of neoplastic lymphocytes (usually B cells) within the lumen of CNS blood vessels but not in the brain parenchyma. In the past, treatment of cIVL with anthracycline-based regimens was unsatisfactory with very short survival times. In the case of CIVL presented here, high-dose methotrexate-based polychemotherapy according to the Bonn protocol plus rituximab therapy was successful and led to a complete clinical and MRI remission which is ongoing 29 months after diagnosis.
\end{abstract}

Keywords: Intravascular lymphoma, Intravascular CNS lymphoma, High-dose methotrexate-based polychemotherapy, Angioendotheliomatosis, Angiotropic lymphoma

\section{Background}

Intravascular lymphoma, also known as intravascular lymphomatosis or angiotropic lymphoma and formerly known as malignant angioendotheliomatosis is a rare neoplastic disorder in which tumour cells are initially confined to the vascular lumen without parenchymal infiltration. While cases of systemic intravascular lymphoma are more frequently encountered, cases of intravascular lymphoma with restricted central nervous system (CNS) involvement (cIVL) are uncommon and only few patients that had been successfully treated have been reported so far [1-4]. We here present a case with a histologically confirmed cIVL that could be successfully treated with a high-dose methotrexate (HD-MTX) and rituximab-based chemotherapy regimen.

\section{Case presentation}

A 69-year-old male Caucasian patient presented with recurrent transient amnestic aphasia and gait ataxia. Physical examination at the time of referral did not reveal

\footnotetext{
* Correspondence: Ulrich.Herrlinger@ukb.uni-bonn.de

${ }^{1}$ Division of Clinical Neurooncology, Department of Neurology, University of Bonn Medical Center, BonnSigmund-Freud-Straße 25, 53127 Bonn, Germany Full list of author information is available at the end of the article
}

any further pathological findings. B symptoms were absent. Serum LDH levels were twice the upper limit of normal, all other serum chemistry and differential blood count was negative. Cerebrospinal fluid (CSF) analysis revealed a normal cell count, protein levels were within the reference range, no atypical cells were detected. Initial magnetic resonance imaging (MRI) revealed a contrastenhancing lesion in the pons (Figure $1 \mathrm{~A}$ ) and additional involvement of the left temporomesial area. A stereotactic biopsy was performed and histology revealed a CD20antigen-expressing intravascular lymphoma with high proliferative activity (Figure 2A, B). Immunohistological evaluation of B-cell differentiation markers showed a BCL-6+ and MUM-1+-status. Subsequent staging (i.e. examination of the chest, abdomen and pelvis by contrast-enhanced computed tomography (CT) scan, bone marrow biopsy, slit lamp examination of the eye, spinal tap) did not reveal any systemic or additional CNS involvement.

Chemotherapy according to the Bonn protocol was initiated in combination with rituximab therapy. The Bonn protocol comprises six 3-week courses with different combinations of HD-MTX ( $3 \mathrm{gm} / \mathrm{m}^{2}$ over 24 hours), ifosfamide, procarbazin, cytarabine, vinca alkaloids, and dexamethasone (for details see [5]). Rituximab was given at

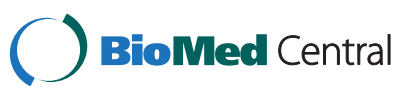




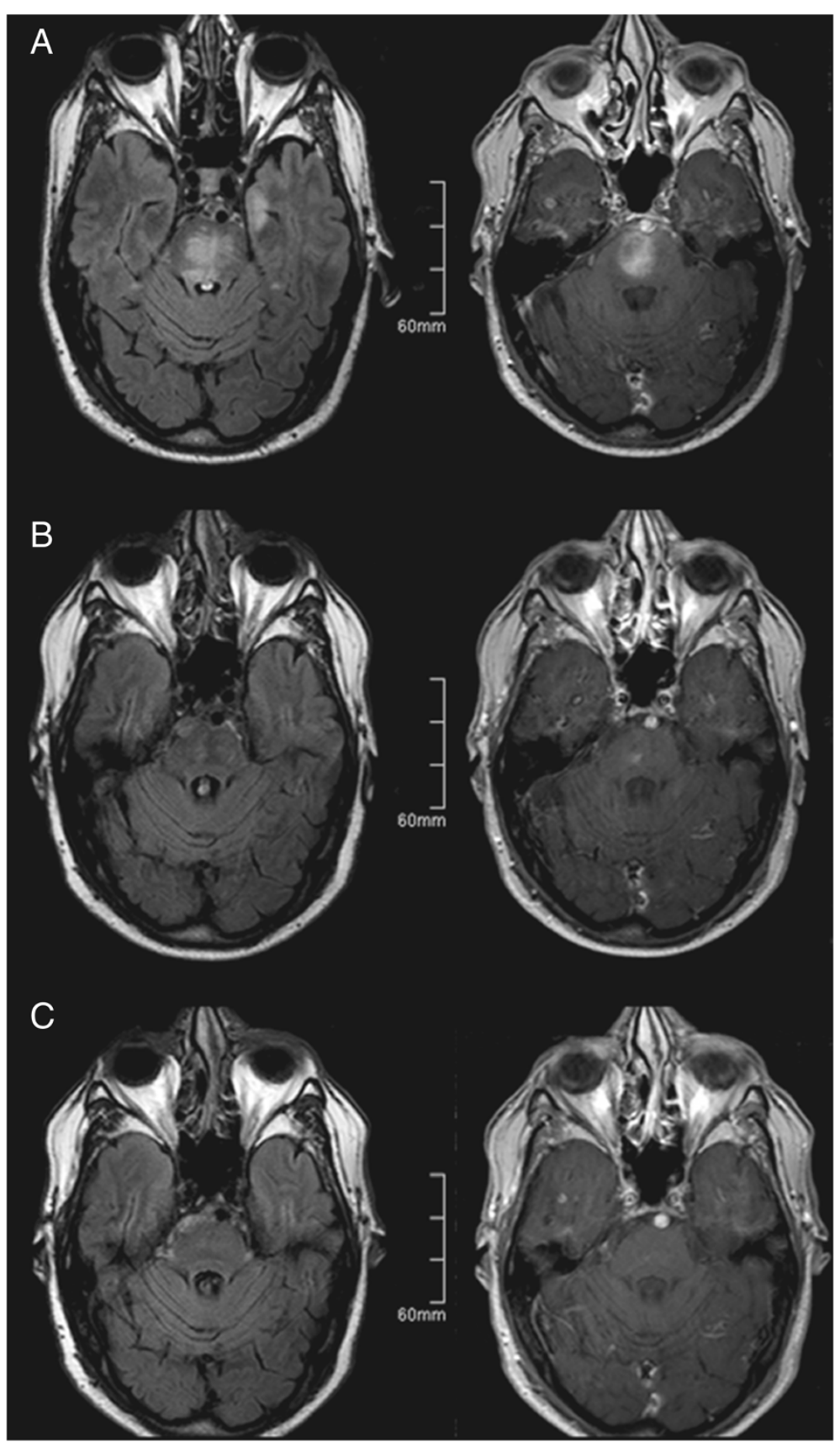

Figure 1 MR imaging prior to and after HD-MTX-based chemotherapy (left column FLAIR, right column: contrast enhanced T1-weighted imaging)MR imaging prior to therapy (A) and at follow-up imaging at the end of 6 courses of chemotherapy with a strong reduction of contrast-enhancing lesions (B). Nineteen months after initiation of treatment MR imaging showed complete regression of marked FLAIR hyperintensities and contrast enhancement in the brain stem (C).

each course one day prior to the start of the HD-MTX infusion. During the 5th course, a transient and moderate increase in serum creatinine occurred, without a need for dose reduction in subsequent treatment courses. Vincristine was removed from the treatment protocol after development of mild signs of polyneuropathy. After the second course, the contrast-enhancing lesion showed already a partial remission; after the sixth course, only one small contrast-enhancing lesion remained that had to be qualified as unconfirmed complete remission since it further diminished in subsequent control MRIs without additional therapy (Figure 1A-C). The patient is now in complete clinical and radiographic remission 29 months after initial diagnosis of cIVL. 

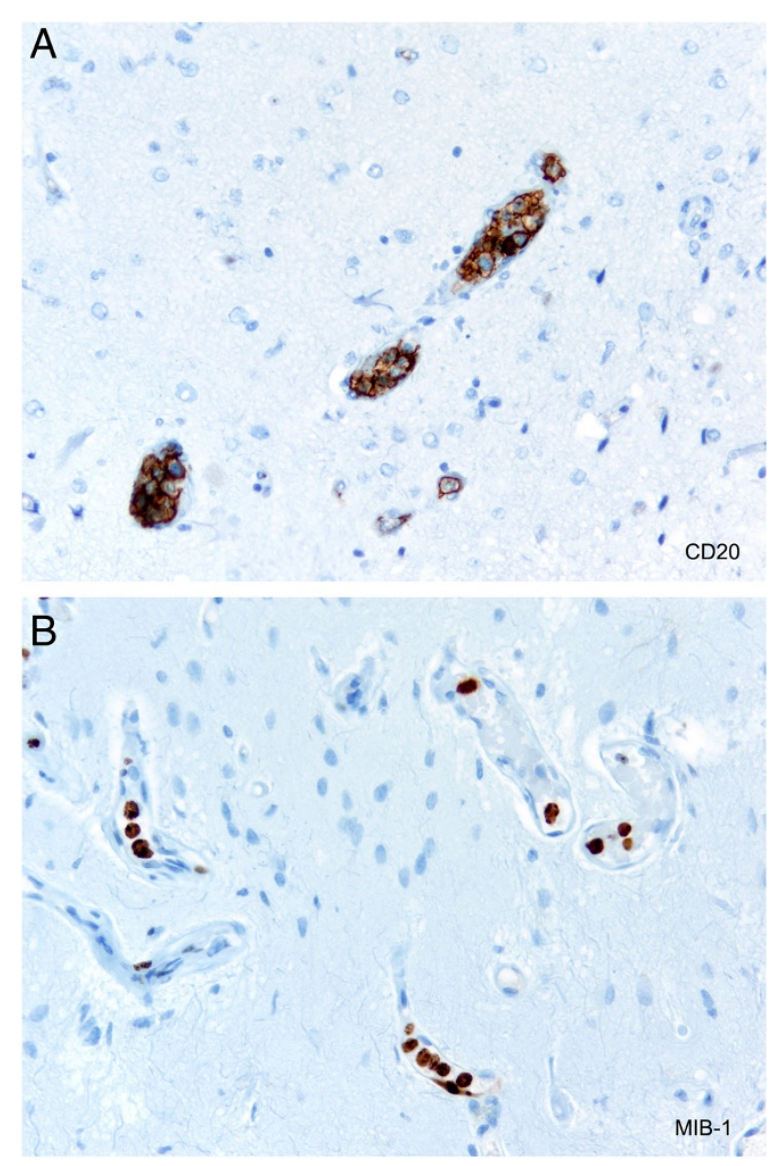

Figure 2 Histological examination of the tissue obtained by stereotactic biopsy of the brain stem. Histology revealed CD20-immunopositive intravascular lymphoma cells (A) with a very high proliferative activity in MIB-1 immunohistochemistry (B)

In this case report we demonstrate the successful therapy of a patient with cIVL, i.e. intravascular lymphoma limited to the CNS. The few reports available on the treatment of this medical condition are summarized in Table 1. All cIVL cases in which progression and death due to systemic failure was explicitly mentioned were not included here. In some cases, lymphoma-directed specific therapy was not applied or the treatment modality was not reported. In these cases, survival did not exceed 4 months [6-9]. Conventional chemotherapy with anthracyline-based protocols (i.e. CHOP in 3 patients), radiotherapy, or corticosteroid therapy was not successful [10-12]. Using anthracycline-based chemotherapy which is effective in systemic intravascular lymphoma does not penetrate the intact blood-brain barrier (BBB), overall survival rarely exceeded 6 months. Our case, on the other hand, is in line with reports demonstrating that BBB-penetrating HD-MTX-based regimens may have considerable efficacy. Seven patients treated with HD-MTX alone or in combination with CHOP survived 6-20 months $[1,13,14]$. In a separate study, three patients with cIVL receiving HD-MTX-based chemotherapy showed progression-free survival times of 2, 20 and
48 month [1-3]. One additional case report presented a patient receiving HD-MTX + R-CHOP followed by consolidation therapy with high-dose chemotherapy (thiotepa, busulfan, and cyclophosphamide) and autologous stem-cell rescue. This patient survived for at least 19 months after treatment [4]. It remains unclear why HDMTX-based, i.e. blood-brain barrier (BBB)-penetrating therapy is needed for successful therapy of cIVL and which are the optimal combination partners for MDMTX therapy. Also, it is unclear why regimens that do not penetrate the BBB but are effective in other forms of intravascular lymphoma are not successful in cIVL. This is particularly puzzling since all cIVL tumour cells are by histological definition located within the vessels and not beyond in the brain parenchyma.

\section{Conclusion}

Overall, on the base of our case and upon reviewing the literature, we recommend the use of HD-MTXbased polychemotherapy similar to HD-MTX-based protocols for primary (parenchymal) CNS lymphoma in patients with cIVL. 
Table 1 Summary of all patients with intravascular lymphomatosis limited to the CNS (cIVL) reported in the literature

\begin{tabular}{|c|c|c|c|c|}
\hline Author & Site of involvement & Neurological symptoms & Treatment & Outcome \\
\hline Baehring et al. [1] & Brain & Right hemiparesis, dysarthria & HD-MTX (induction 5, consolidation 10, maintenance 2) & CR 20 months after diagnosis \\
\hline Baehring et al. [1] & Brain, spinal cord & Proximal spastic paraparesis, psychosis & HD-MTX (induction 12) & PR 18 months after diagnosis \\
\hline Baehring et al. [1] & Brain, nerve roots & Dysarthria, gait disturbance, allodynia & $\begin{array}{l}\text { HD-MTX initially (induction 6, consolidation 4), HD-MTX } \\
\text { salvage (6 induction, } 7 \text { consolidation) }\end{array}$ & $\begin{array}{l}\text { PR until } 8 \text { months after diagnosis: PR } \\
\text { until } 12 \text { months after recurrence }\end{array}$ \\
\hline Baehring et al. [1] & Brain & Cognitive decline, homonymous hemianopsia, ataxia & HD-MTX (induction 1 cycle) & $\begin{array}{l}\text { Died of disease progression after first } \\
\text { cycle of chemotherapy }\end{array}$ \\
\hline Calamia et al. [15] & CNS & NA & m-BACOD & OS 16 months \\
\hline Calamia et al. [15] & CNS & NA & Pro-MACE-CytaBOM & OS 44 months \\
\hline Bergmann et al. [6] & Brain & Left-sided hemiparesis & NA & OS 2 months \\
\hline Bergmann et al. [6] & Brain & Spastic paraparesis, left arm paresis & NA & OS 2 months \\
\hline DiGiuseppe et al. [3] & Brain & Mental status changes & $\begin{array}{l}\text { Pro-MACE-CytaBOM, ifosfamide/NP-16/cisplatin \& whole } \\
\text { brain irradiation ( } 45 \text { Gy) }\end{array}$ & CR 48 months after diagnosis \\
\hline Kanda et al. [16] & CNS & Aphasia, apraxia & CHOP, VEMP, radiotherapy & OS one month \\
\hline Aznar et al. [7] & CNS & Distal paresthesia of the lower limbs, paraparesis & NA & OS few months \\
\hline Passarin [8] & Brain & Progressive cognitive deterioration, tetraparesis & NA & OS 3-4 weeks \\
\hline Natali-Sora et al. [17] & CNS & Generalized tonic-clonic seizures & $\begin{array}{l}\text { Cyclophosphamide, mitoxantrone, BCNU, } \\
\text { methylprednisolone }\end{array}$ & CR 46 months after diagnosis \\
\hline Liow et al. [18] & CNS & NA & $\mathrm{CHOP}$ & OS 13 months \\
\hline Albrecht et al. [9] & Brain & Cognitive deterioration, aphasia & NA & OS few weeks \\
\hline Ferreri et al. [10] & CNS & NA & CHOP (3 patients), CVP (one patient) & OS less than 4 months \\
\hline Holmøy et al. [12] & Brain & vertigo, diplopia, left-sided hearing loss, aphasia & high-dose corticosteroid pulse therapy & OS 18 weeks \\
\hline Momota et al. [14] & Brain & Left-sided hemiparesis & HD-MTX, whole brain irradiation & OS 6 months \\
\hline This case & Brain & Transient amnestic aphasia, gait ataxia & Bonn protocol + rituximab & CR 29 months after diagnosis \\
\hline
\end{tabular}

Abbreviations: HD-MTX: high dose methotrexate; CR: complete remssion; PR: partial response; NA: not available; OS: overall survival; m-BACOD: cyclophosphamide, doxorubicin, vincristine, bleomycin, dexamethasone, methotrexate; Pro-MACE-CytaBOM: prednisone, methotrexate (with leucovorin rescue), doxorubicin, cyclophosphamide, etoposide, cytarabine, bleomycin, vincristine; VP-16: etoposide; CHOP: cyclophosphamide, doxorubicin, vincristine, prednisolone; VEMP: vincristine, cyclophosphamide, mercaptopurine, prednisolone; CVP: cyclophosphamide, vincristine and prednisone; Bonn protocol: HD-MTX, ifosfamide, procarbazin, cytarabine, vinca alkaloids, dexamethasone. 


\section{Consent}

Written informed consent was obtained from the patient for publication of this Case report and any accompanying images. A copy of the written consent is available for review by the Editor-in-Chief of this journal.

\section{Competing interests}

The authors declare that they have no competing interests.

\section{Authors' contributions}

Conceived and designed therapy: UH MG. Performed neuroradiologic analysis: HU MN. Neuropathological diagnosis: KK PN. Wrote the paper: SK UH. Performed treatment and participated in collecting data: SK YK ST MS FM NS. All authors read and approved the final manuscript.

\section{Author details}

'Division of Clinical Neurooncology, Department of Neurology, University of Bonn Medical Center, BonnSigmund-Freud-Straße 25, 53127 Bonn, Germany 2 Department of Radiology, University of Bonn Medical Center, BonnSigmund-Freud-Straße 25, 53127 Bonn, Germany. ${ }^{3}$ Department of Neuropathology, University of Bonn Medical Center, BonnSigmund-Freud-Straße 25, 53127 Bonn, Germany. ${ }^{4}$ Department of Oncology, University of Bonn Medical Center, BonnSigmund-Freud-Straße 25, 53127 Bonn, Germany. ${ }^{5}$ Stem Cell Pathologies, Institute of Reconstructive Neurobiology, University of Bonn Medical Center,

BonnSigmund-Freud-Straße 25, 53127 Bonn, Germany.

Received: 25 October 2012 Accepted: 24 November 2012

Published: 5 December 2012

\section{References}

1. Baehring JM, Longtine J, Hochberg FH: A new approach to the diagnosis and treatment of intravascular lymphoma. J Neurooncol 2003, 61:237-48.

2. Debiais S, Bonnaud I, Cottier JP, et al: A spinal cord intravascular lymphomatosis with exceptionally good outcome. Neurology 2004, 63:1329-30.

3. DiGiuseppe JA, Nelson WG, Seifter EJ, Boitnott JK, Mann RB: Intravascular lymphomatosis: a clinicopathologic study of 10 cases and assessment of response to chemotherapy. J Clin Oncol 1994, 12:2573-9.

4. Pless ML, Chen YB, Copen WA, Frosch MP: Case records of the Massachusetts General Hospital. Case 9-2010. A 37-year-old woman with paresthesias and ataxia. N Engl J Med 2010, 362:1129-38.

5. Pels H, Schmidt-Wolf IG, Glasmacher A, et al: Primary central nervous system lymphoma: results of a pilot and phase II study of systemic and intraventricular chemotherapy with deferred radiotherapy. J Clin Oncol 2003, 21:4489-95.

6. Bergmann M, Terzija-Wessel U, Blasius $\mathrm{S}$, et al: Intravascular lymphomatosis of the CNS: clinicopathologic study and search for expression of oncoproteins and Epstein-Barr virus. Clin Neurol Neurosurg 1994, 96:236-43.

7. Aznar AO, Montero MA, Rovira R, Vidal FR: Intravascular large B-cell lymphoma presenting with neurological syndromes: clinicopathologic study. Clin Neuropathol 2007, 26:180-6.

8. Passarin MG, Wen PY, Vattemi E, et al: Intravascular lymphomatosis and intracerebral haemorrhage. Neuro/ Sci 2010, 31:793-7.

9. Albrecht R, Krebs B, Reusche E, Nagel M, Lencer R, Kretzschmar HA: Signs of rapidly progressive dementia in a case of intravascular lymphomatosis. Eur Arch Psychiatry Clin Neurosci 2005, 255:232-5

10. Ferreri AJ, Campo E, Ambrosetti A, et al: Anthracycline-based chemotherapy as primary treatment for intravascular lymphoma. Ann Oncol 2004 15:1215-21

11. Glass J, Hochberg FH, Miller DC: Intravascular lymphomatosis: A systemic disease with neurologic manifestations. Cancer 1993, 71:3156-64.

12. Holmøy T, Nakstad PH, Fredø HL, et al: Intravascular large B-cell lymphoma presenting as cerebellar and cerebral infarction. Arch Neurol 2007, 64:754-5.

13. Moussouttas M: Intravascular lymphomatosis presenting as posterior leukoencephalopathy. Arch Neurol 2002, 59:640-1.

14. Momota H, Narita Y, Miyakita Y, et al: Intravascular lymphoma of the central nervous system presenting as multiple cerebral infarctions. Nagoya J Med Sci 2012, 74:353-8.
15. Calamia KT, Miller A, Shuster EA, Perniciaro C, Menke DM: Intravascular lymphomatosis. A report of ten patients with central nervous system involvement and a review of the disease process. Adv Exp Med Biol 1999, 455:249-65.

16. Kanda M, Suzumiya J, Ohshima K, Tamura K, Kikuchi M: Intravascular large cell lymphoma: clinicopathological, immuno-histochemical and molecular genetic studies. Leuk Lymphoma 1999, 34:569-80.

17. Natali-Sora MG, Lodi M, Corbo M, Hays AP, Nemni R: Intravascular malignant lymphomatosis with neurological symptoms. J Neurol 1996, 243:205-6

18. Liow $K$, Asmar $P$, Liow M, et al: Intravascular lymphomatosis: contribution of cerebral MRI findings to diagnosis. J Neuroimaging 2000, 10:116-8.

doi:10.1186/2162-3619-1-37

Cite this article as: Kebir et al.: Intravascular CNS lymphoma: Successful therapy using high-dose methotrexate-based polychemotherapy. Experimental Hematology \& Oncology 2012 1:37.

\section{Submit your next manuscript to BioMed Central and take full advantage of:}

- Convenient online submission

- Thorough peer review

- No space constraints or color figure charges

- Immediate publication on acceptance

- Inclusion in PubMed, CAS, Scopus and Google Scholar

- Research which is freely available for redistribution 